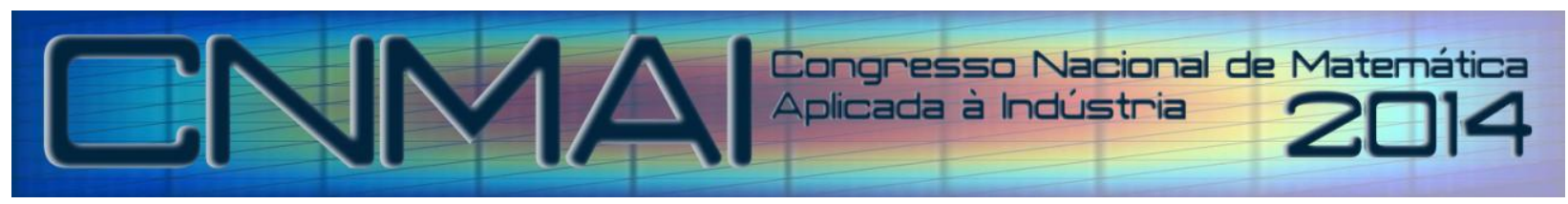

18 a 21 de novembro de 2014, Caldas Novas - Goiás

\title{
ESTUDO DE UMA NOVA ESTRUTURA ROBÓTICA PARALELA COMBINADA
}

\author{
João Marcus Teixeira Pêgo, j.marcus.gtr7@ hotmail.com ${ }^{1}$ \\ João Carlos Mendes Carvalho, jcmendes@mecanica.ufu.br ${ }^{1}$ \\ Rogério Sales Gonçalves, rsgoncalves@mecanica.ufu.br ${ }^{1}$
}

${ }^{1}$ Universidade Federal de Uberlândia - Faculdade de Engenharia Mecânica - Av. João Naves de Ávila, 2121, Bairro Santa Mônica, Uberlândia - MG - CEP 38400-902

\begin{abstract}
Resumo: As estruturas robóticas são comumente divididas em estruturas seriais, paralelas e híbridas. A estrutura serial consiste de um elemento terminal, ligado a uma base por meio de uma cadeia cinemática única. Uma estrutura paralela por sua vez pode ser descrita como um mecanismo formado por um elemento terminal, ligado a uma base fixa por meio de duas ou mais cadeias cinemáticas. As estruturas paralelas podem ter algumas vantagens sobre as estruturas seriais, como melhores índices de rigidez e capacidade maior de carga, porém sofrem com um espaço de trabalho limitado e maior complexidade na modelagem matemática. O terceiro tipo de estruturas são as híbridas, combinações das estruturas seriais com as estruturas paralelas. Dentro das estruturas hibridas existem também combinações entre estruturas paralelas. Desta forma este trabalho apresenta uma nova estrutura robótica paralela combinada. Esta é formada pelo mecanismo $5 R$ plano simétrico e o mecanismo esférico paralelo. $O$ modelo matemático desta nova estrutura é calculado através dos modelos individuais das estruturas que a compõem. Finalmente, foi desenvolvido o modelo gráfico tridimensional da nova estrutura robótica paralela combinada e confrontado com o modelo matemático.
\end{abstract}

Palavras-chave: Estruturas Robóticas, Mecanismo 5R Plano, Mecanismo Esférico Paralelo.

\section{INTRODUÇÃO}

As estruturas robóticas são comumente divididas em estruturas serial, paralela e híbrida.

A estrutura serial consiste de um elemento terminal ou manipulador, ligado a uma base por meio de uma cadeia cinemática única, fornecendo assim um percurso único para ir da base até o elemento terminal e retornar à base, num trajeto de ida e volta passando pelos mesmos elementos (Gonçalves, 2009).

Uma estrutura paralela por sua vez pode ser descrita como um mecanismo formado por um elemento terminal ou plataforma móvel, ligado a uma base fixa por meio de duas ou mais cadeias cinemáticas independentes. As estruturas paralelas têm algumas vantagens sobre as estruturas seriais, como poder ter maior rigidez e quantidade de carga suportada por peso, porém sofrem com um espaço de trabalho limitado. Assim, em situações onde alta precisão, velocidade e rigidez são mais importantes que o espaço de trabalho, as estruturas paralelas são uma alternativa às estruturas seriais (Tanev, 2000a).

O terceiro tipo de estruturas são as híbridas, combinações das estruturas seriais com as estruturas paralelas. Dentre as estruturas híbridas existe também a combinação de estruturas paralelas. Estas estruturas serão denominadas neste trabalho de estruturas paralelas combinadas. Estas estruturas podem superar o problema do espaço de trabalho limitado, e fornecerem vantagens tanto das estruturas serial quanto paralela (Zheng et al., 2004; Zhao et al., 2009).

$\mathrm{O}$ estudo das estruturas paralelas combinadas pode ser dividido em função da disposição das cadeias paralelas em três tipos de combinação: serial com as estruturas paralelas ligadas em sequencia (Ramadan et al., 2009; Tanev, 2006; Tanikawa et al., 1995; 1996; Wu et al., 2008; Zheng et al., 2004; Yabugaki et al., 2013); ligação em paralelo das cadeias paralelas (Yoon e Ryu, 2005); e misto que é a combinação de estruturas paralelas superpostas em série e paralelo (Badescu e Mavroidis, 2004; Badescu et al., 2002).

As diversas estruturas híbridas propostas na literatura apresentam vantagem de expandir o espaço de trabalho da estrutura paralela podendo também aumentar a sua acuracidade e rigidez. Estas são utilizadas com a capacidade de se 
reconfigurar (Badescu et al., 2002), simular com riqueza de detalhes o caminhar humano (Yoon e Ryu, 2005) e trabalhar em locais de difícil acesso ou grande perigo para o homem (Wu et al., 2008), tornando o estudo desse novo modelo de estrutura, extremamente promissor.

Desta forma este trabalho apresenta o desenvolvimento de uma nova estrutura paralela combinada. Esta é formada pelo mecanismo 5R plano simétrico e o mecanismo esférico paralelo. O modelo matemático desta nova estrutura é calculado através dos modelos individuais das estruturas que a compõem. Finalmente, foi desenvolvido o modelo gráfico tridimensional da nova estrutura robótica paralela combinada e confrontado com o modelo matemático.

\section{ESTRUTURA PARALELA COMBINADA PROPOSTA}

Neste item será apresentada a modelagem matemática de uma nova estrutura paralela combinada. Esta nova estrutura é formada pelo manipulador paralelo simétrico $5 \mathrm{R}$ e o manipulador esférico paralelo. $\mathrm{O}$ modelo matemático desta estrutura é obtido a partir dos modelos individuais de cada estrutura.

\subsection{Manipulador Paralelo Simétrico 5R}

O mecanismo de 5 barras, também conhecido como mecanismo 5R é um típico mecanismo paralelo com o mínimo de graus de liberdade, que pode ser utilizado para posicionar um ponto em uma região de um plano. Este consiste de 5 barras conectadas entre si por 5 articulações de rotação, duas das quais conectadas à base são atuadas, Fig. 1 (Liu et. al, 2006b).

A escolha desta estrutura para compor a estrutura paralela combinada foi realizada por permitir o movimento plano com dois graus de liberdade (translações em x e y), Fig. 1, por ser uma estrutura relativamente simples e amplamente estudada. Assim, o mecanismo 5R será à base do manipulador combinado, sendo responsável pela posição no plano do manipulador combinado.

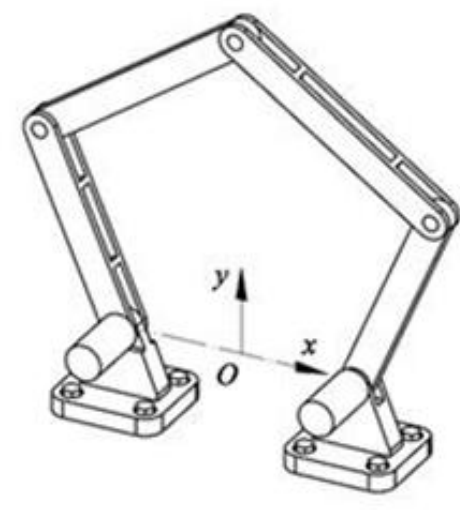

(a)

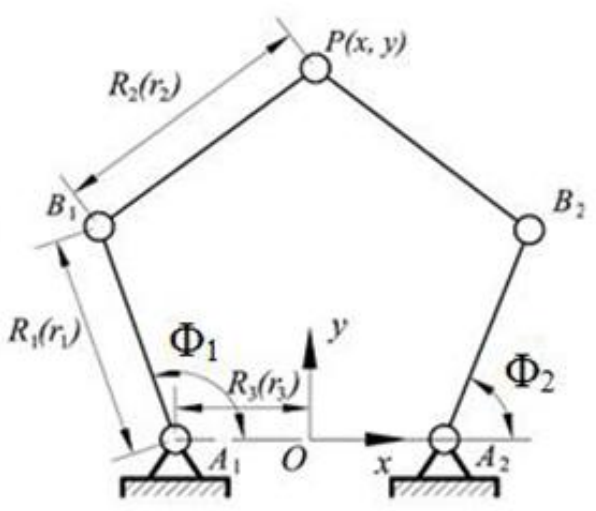

(b)

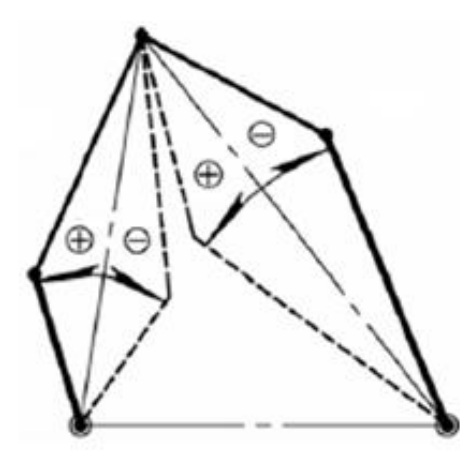

(c)

Figura 1. (a) Manipulador Paralelo 5R; (b) esquema cinemático; (c) Configurações do Manipulador 5R

(Liu et. al, 2006b).

\subsection{Manipulador Esférico Paralelo e o Agile Eye}

Um manipulador esférico paralelo é geralmente composto por duas plataformas em forma de pirâmide, a saber, a plataforma base e a plataforma móvel, que são conectadas por três segmentos igualmente espaçados, contendo somente articulações de rotação. Os eixos de todas as articulações se interceptam num ponto comum, chamado de centro de rotação da estrutura. O movimento da plataforma móvel fica confinado à superfície de uma esfera centrada no centro de rotação, resultando em três graus de liberdade compostos apenas de rotações (Bai, 2010).

A maioria das aplicações de mecanismos esféricos paralelos pode ser encontrada em dispositivos de orientação, como articulações de rotação, orientação de ferramentas médicas (Li e Payandeh, 2002) e dispositivos de orientação de câmera, como por exemplo, o Agile Eye, Fig. 2(a) (Gosselin e Hamel, 1994; Gosselin et. al. 1996).

O Agile Eye é um manipulador esférico com formato simétrico e sua configuração favorece o espaço de trabalho, com segmentos como os da Fig. 2(b), que visam melhorar o desempenho e minimizar as interferências do mecanismo. Este foi escolhido como modelo para acrescentar os 3 graus de liberdade de orientação (3 rotações) à estrutura robótica paralela combinada proposta neste trabalho. 


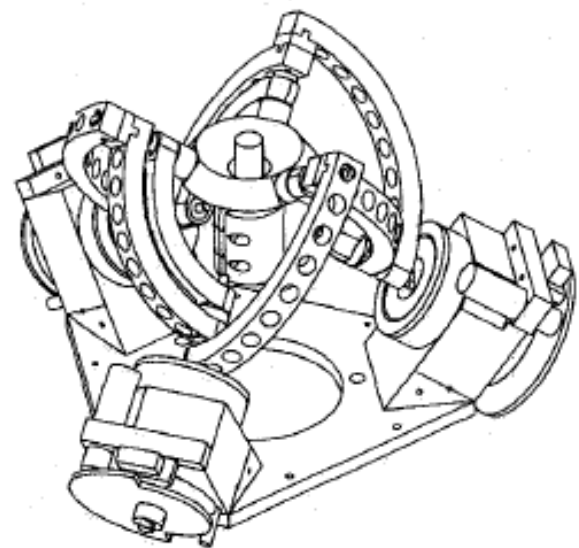

(a)

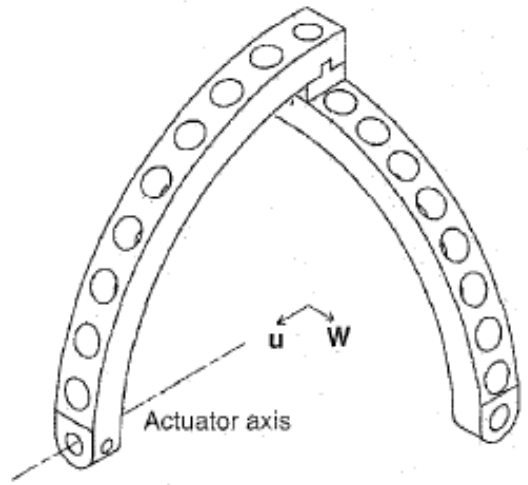

(b)

Figura 2. (a) Modelo do Agile Eye (Gosselin E Hamel, 1994); (b) Segmento proximal de cada perna do mecanismo (Gosselin et. al. 1996).

\section{MODELO CINEMÁTICO DO MANIPULADOR PARALELO COMBINADO}

Neste item é desenvolvido o modelo cinemático inverso para o manipulador combinado proposto, que será uma combinação das duas estruturas apresentadas no item 2. Primeiro são calculados os ângulos dos atuadores do mecanismo esférico paralelo (que definem a orientação) e em seguida são encontrados os ângulos dos atuadores do mecanismo 5R (que definem a posição).

\subsection{Modelo cinemático do Manipulador Esférico.}

A Figura 3(a) apresenta um manipulador esférico paralelo genérico. O manipulador consiste somente de articulações de rotação, cujos eixos são denotados pelos vetores unitários ui, vi, e wi. Os três segmentos (vermelhos) conectados à plataforma base tem dimensões idênticas $\alpha 1$, enquanto os três segmentos (verdes) que são conectados à plataforma móvel tem dimensões idênticas $\alpha 2$. Além disto, $\beta$ e $\gamma$ definem a geometria de duas pirâmides regulares da base e da plataforma móvel. A articulação esférica ativa é um caso especial de manipulador esférico paralelo para o qual $\gamma=0$ (Bai, 2010).

Para a estrutura Agile Eye, tem-se um caso específico onde $\beta=\gamma$ (Gosselin e Hamel, 1994; Gosselin et. al. 1996), e os parâmetros de construção são:

$$
\alpha_{1}=90^{\circ} ; \alpha_{2}=90^{\circ} ; \beta=\gamma=54.74^{\circ} \text { e } n=45.34 \mathrm{~mm} \text {. }
$$

Sendo $n$ o módulo do vetor n, Fig. 3(a).
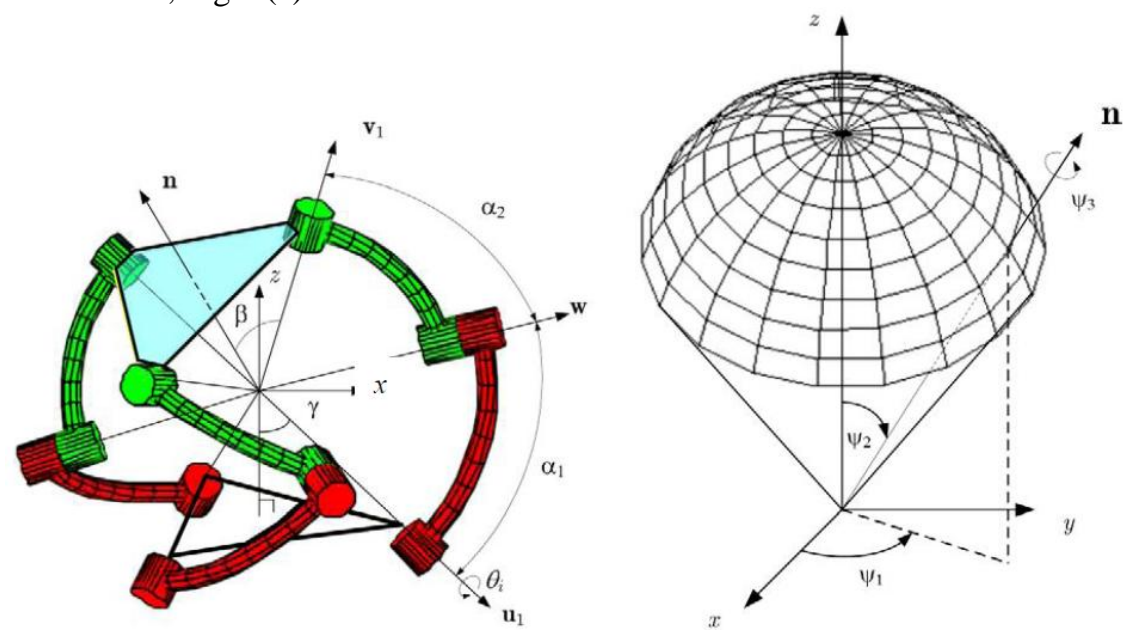

Figura 3. Modelo cinemático de um manipulador esférico paralelo: (a) convenção de simbologia e (b) espaço de trabalho descrito (Bai, 2010).

Um Sistema de coordenadas é escolhido para o manipulador esférico paralelo, com origem localizada no centro de rotação do mecanismo, de forma que o eixo $\mathrm{z}$ forme o mesmo ângulo com todos os vetores $\mathbf{u}_{i}, i=1,2,3$, e de forma que o vetor $\boldsymbol{u}_{l}$ esteja localizado no plano XZ conforme Fig. 3. 
A partir da Fig. 3(b), a orientação do mecanismo esférico paralelo é descrita por um vetor de ângulos $\psi=\left[\psi_{1}, \psi_{2}\right.$, $\left.\psi_{3}\right]^{\mathrm{T}}$ para o qual a matriz de rotação é definida por duas rotações sequenciais (Bai, 2010), Eq. (2).

$$
\mathbf{Q}=\operatorname{rot}\left(\mathbf{e}_{2}, \psi_{2}\right) * \operatorname{rot}\left(\mathbf{e}_{3}, \psi_{3}\right)
$$

Onde $\mathbf{e}_{2}=\left[-\operatorname{sen} \psi_{1}, \cos \psi_{1}, 0\right]^{\mathrm{T}}, \mathbf{e} 3=[0,0,1]^{\mathrm{T}}$ e $\operatorname{rot}(\mathbf{e}, \psi)$ é uma matriz de rotação seguindo a representação ânguloeixo. Assim, as matrizes de rotação podem ser escritas conforme Eqs. (3) e (4).

$$
\begin{gathered}
\operatorname{rot}\left(\boldsymbol{e}_{2}, \psi_{2}\right)=\left[\begin{array}{ccc}
\cos \psi_{2}-\operatorname{sen}^{2} \psi_{1} *\left(\cos \psi_{2}-1\right) & \cos \psi_{1} * \operatorname{sen} \psi_{1} *\left(\cos \psi_{2}-1\right) & \cos \psi_{1} * \operatorname{sen} \psi_{2} \\
\cos \psi_{1} * \operatorname{sen} \psi_{1} *\left(\cos \psi_{2}-1\right) & \cos \psi_{2}-\cos ^{2} \psi_{1} *\left(\cos \psi_{2}-1\right) & \operatorname{sen} \psi_{1} * \operatorname{sen} \psi_{2} \\
-\cos \psi_{1} * \operatorname{sen} \psi_{2} & -\operatorname{sen} \psi_{1} * \operatorname{sen} \psi_{2} & \cos \psi_{2}
\end{array}\right] \\
\operatorname{rot}\left(\boldsymbol{e}_{3}, \psi_{3}\right)=\left[\begin{array}{ccc}
\cos \left(\psi_{3}\right) & -\operatorname{sen}\left(\psi_{3}\right) & 0 \\
\operatorname{sen}\left(\psi_{3}\right) & \cos \left(\psi_{3}\right) & 0 \\
0 & 0 & 1
\end{array}\right]
\end{gathered}
$$

No Sistema de coordenadas escolhido, o vetor unitário $\mathbf{u}_{i}$, Fig. 3(a), é dado pela Eq. (5)

$$
\mathbf{u}_{i}=\left[\begin{array}{c}
\cos \eta_{i} \operatorname{sen} \gamma \\
\operatorname{sen} \eta_{i} \operatorname{sen} \gamma \\
-\cos \gamma
\end{array}\right]
$$

Onde $\eta i=2(i-1) \pi / 3$.

$\mathrm{O}$ vetor unitário $\mathbf{w}_{i}, i=1,2,3$ do eixo da articulação de rotação intermediária do $i$-ésimo segmento é obtido em termos do ângulo de entrada $\theta_{i}, i=1,2,3$ e dos parâmetros de construção do mecanismo como:

$$
\boldsymbol{w}_{i}=\left[\begin{array}{c}
s \alpha_{1} *\left(s \eta_{i} * s \theta_{i}+c \gamma * c \eta_{i} * c \theta_{i}\right)+c \alpha_{1} * c \eta_{i} * s \gamma \\
c \alpha_{1} * s \gamma * s \eta_{i}-s \alpha_{1} *\left(c \eta_{i} * s \theta_{i}-c \gamma * c \theta_{i} * s \eta_{i}\right) \\
s \alpha_{1} * c \theta_{i} * s \gamma-c \alpha_{1} * c \gamma
\end{array}\right]
$$

Onde na Eq. (6) denota-se $s$ para seno, e $c$ para cosseno.

$\mathrm{O}$ vetor unitário $\boldsymbol{v}_{i}$, Eq. (7), paralelo ao eixo da articulação superior de rotação do $i$-ésimo segmento é uma função da orientação da plataforma móvel. Sendo esta orientação descrita pela matriz de rotação $\mathbf{Q}$.

$$
\mathbf{v}_{i}=\mathbf{Q} \mathbf{v}_{i}{ }^{*}
$$

Onde $\mathbf{v}_{i}$ *é o vetor unitário $\mathbf{v}_{\mathrm{i}}$, Fig. 3(a), para o eixo da articulação superior de rotação do $i$-ésimo segmento quando a plataforma móvel está em sua orientação de referência, que pode ser escrito conforme Eq. (8).

$$
\mathbf{v}_{i}^{*}=\left[\begin{array}{c}
\cos \eta_{i} \operatorname{sen} \gamma \\
\operatorname{sen} \eta_{i} \operatorname{sen} \gamma \\
\cos \gamma
\end{array}\right]
$$

Para a cadeia fechada do manipulador esférico paralelo, Fig. 3(a), a seguinte equação é obtida:

$$
\mathbf{w}_{i} \cdot \mathbf{v}_{i}=\cos \alpha_{2}, i=1,2,3
$$

Substituindo as Eqs. (7) e (6) na Eq. (9), obtém-se:

$$
A_{i} t_{i}^{2}+2 B_{i} t_{i}+C_{i}=0, i=1,2,3
$$

Onde os coeficientes $A_{i}, B_{i}, e C_{i}$, são funções das coordenadas cartesianas e dos parâmetros geométricos do robô, conforme Eq. (11).

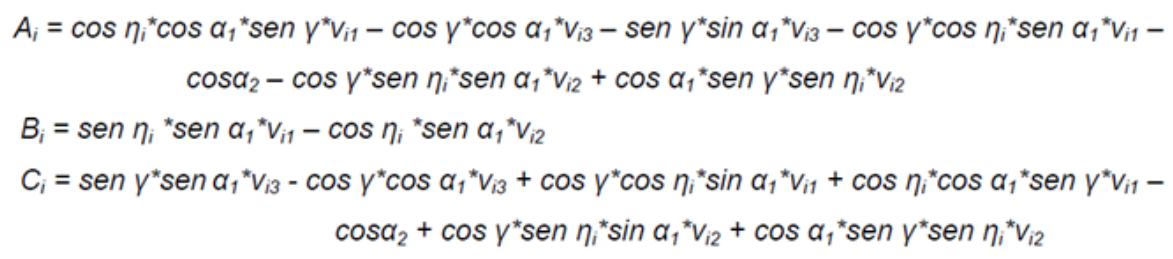


Sendo $\mathrm{v}_{i j}, j=1,2,3, \mathrm{o} j$-ésimo, termo do vetor $\boldsymbol{v}_{i}$.

A solução para o problema da cinemática inversa do manipulador esférico paralelo é obtida pela resolução da equação de segundo grau, Eq. (10), para cada segmento do manipulador, Eq. (12).

$$
t_{i}=\frac{-B_{i} \pm \sqrt{B_{i}^{2}-A_{i} C_{i}}}{A i}, i=1,2,3
$$

Fornecendo assim dois valores para cada $t_{i}, i=1,2,3$, consequentemente dois valores para cada $\theta_{i}$, o que resulta em oito possíveis soluções para a orientação do mecanismo esférico paralelo.

\subsection{Modelo cinemático do manipulador paralelo $5 \mathbf{R}$.}

O modelo cinemático do manipulador 5R pode ser desenvolvido a partir dos parâmetros representados na Fig. 1(b). Cada articulação ativa é chamada $\mathrm{A}_{i}(i=1,2)$, a outra extremidade do segmento atuado é chamada $\mathrm{B}_{i}$ e a articulação comum aos dois segmentos é denotada como $P$, que é também o ponto de saída do mecanismo.

Um sistema de coordenadas global $O x y$ se encontra no centro de $A_{l} A_{2}$ sendo o eixo $y$ normal à $A_{1} A_{2}$ e o eixo $x$ dirigido ao longo de $A_{1} A_{2}$. Para o manipulador 5R simétrico, tem-se $O A_{1}=O A_{2}, A_{1} B_{1}=A_{2} B_{2}$ e $B_{1} P=B_{2} P$. O comprimento do segmento ativo para cada perna é denotado como $A_{i} B_{i}=r_{1}$. Adicionalmente, $B_{i} P=r_{2}$ e $O A_{i}=r_{3}$.

A posição do ponto de saída $P$ no sistema de coordenadas $O x y$ pode ser descrita como a posição do vetor p, Eq. (13).

$$
\mathbf{p}=\left(\begin{array}{l}
x \\
y
\end{array}\right)
$$

No sistema de coordenadas $O x y$, a posição dos vetores $\mathbf{b}_{k}$ dos pontos $B_{k}$ podem ser escritos conforme Eq. (14).

$$
\begin{aligned}
& \mathbf{b}_{1}=\left[\begin{array}{c}
r_{1} \cos \Phi_{1}-r_{3} \\
r_{1} \operatorname{sen} \Phi_{1}
\end{array}\right] \\
& \mathbf{b}_{2}=\left[\begin{array}{c}
r_{1} \cos \Phi_{2}+r_{3} \\
r_{1} \operatorname{sen} \Phi_{2}
\end{array}\right]
\end{aligned}
$$

Onde $\Phi_{1}$ e $\Phi_{2}$ são os ângulos de entrada dos dois segmentos. Então, o problema da cinemática inversa pode ser resolvido a partir da Eq. (15) de restrição:

$$
\left|\mathbf{p b}_{k}\right|=r_{2}, k=1,2
$$

Ou em outra forma, desenvolvendo a Eq. (15):

$$
\begin{aligned}
& \left(x-r_{1} \cos \Phi_{1}+r_{3}\right)^{2}+\left(y-r_{1} \operatorname{sen} \Phi_{1}\right)^{2}=r_{2}{ }^{2} \\
& \left(x-r_{1} \cos \Phi_{2}-r_{3}\right)^{2}+\left(y-r_{1} \operatorname{sen} \Phi_{2}\right)^{2}=r_{2}{ }^{2}
\end{aligned}
$$

Se a posição do ponto de saída $P$ é conhecida, as entradas (coordenadas generalizadas) para se atingir tal posição podem ser encontradas através da equação de segundo grau, Eq. (17), obtida a partir da Eq. (16).

$$
a_{k} z_{k}^{2}+b_{k} z_{k}+c_{k}=0
$$

Onde:

$$
z_{k}=\frac{-b_{k} \pm \sqrt{b_{k}^{2}-4 a_{k} c_{k}}}{2 a_{k}}, k=1,2
$$

E os termos que solucionam a Eq. (18) são dados na Eq. (19). 


$$
\begin{aligned}
& a_{1}=r_{1}^{2}+y^{2}+\left(x+r_{3}\right)^{2}-r_{2}^{2}+2\left(x+r_{3}\right) r_{1} \\
& b_{1}=-4 y r_{1} \\
& c_{1}=r_{1}^{2}+y^{2}+\left(x+r_{3}\right)^{2}-r_{2}^{2}-2\left(x+r_{3}\right) r_{1} \\
& a_{2}=r_{1}^{2}+y^{2}+\left(x-r_{3}\right)^{2}-r_{2}^{2}+2\left(x-r_{3}\right) r_{1} \\
& \quad b_{2}=b_{1}=-4 y r_{1} \\
& \quad c_{2}=r_{1}^{2}+y_{2}+\left(x-r_{3}\right)^{2}-r_{2}^{2}-2\left(x-r_{3}\right) r_{1} .
\end{aligned}
$$

Substituindo os termos da Eq. (19) na Eq. (18), encontram-se quatro soluções para o problema da cinemática inversa do manipulador 5R. A configuração mostrada na Fig. 1(a) pode ser obtida se o sinal “ \pm ” na Eq. (18) é “+” para o caso de $i=1$ e é "-" para o caso $i=2$. Tal configuração é denotada como modelo "+-". Há outros três modelos então, que são os modelos “-+”, “-_", e “++”, respectivamente. Estes quatro modelos cinemáticos inversos correspondem a quatro formas de trabalho do mecanismo, que podem ser vistas na Fig. 1(c) (Liu et. al 2006b).

Neste trabalho foi utilizada a estrutura em sua configuração "+-" com parâmetros de construção de: $r_{1}=r_{2}=480$ $m \mathrm{~m}$ e $r_{3}=240 \mathrm{~mm}$.

Considerando os ângulos de orientação $\psi_{1}, \psi_{2}$ e $\psi_{3}$, da estrutura esférica e o sistema de coordenadas na origem do manipulador paralelo 5R, conforme Fig. 1(b), e o módulo do vetor $\mathbf{n}$ do manipulador esférico paralelo, Fig. 3(a), podese definir as coordenadas $x$ e $y$ do ponto $\mathrm{P}$.

A ligação entre os dois mecanismos é feita através do ponto $\mathrm{P}$ do mecanismo $5 \mathrm{R}$ e do centro da base do mecanismo esférico paralelo. Assim, é possível projetar o elemento terminal do mecanismo esférico paralelo no plano que contém o mecanismo 5R, conforme Fig. 4, obtendo assim o ponto N.

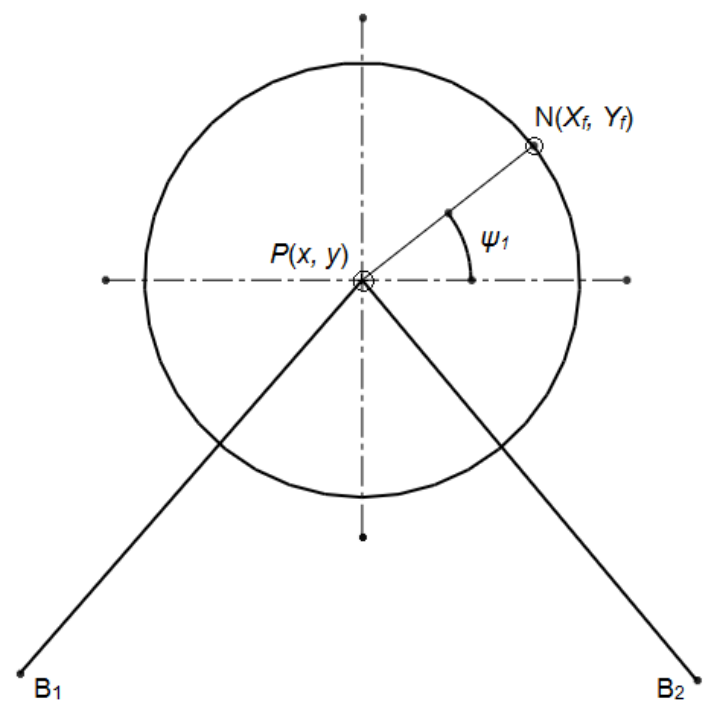

Figura 4. Projeção do elemento terminal do Mecanismo Esférico Paralelo no plano XY.

O segmento PN pode ser escrito como $n^{*} \operatorname{sen}(\psi 2)$. Assim, sendo $X_{f}$ e $Y_{f}$ as coordenadas de saída do elemento terminal do Mecanismo Esférico Paralelo em relação à origem $\mathrm{O}$ da Fig. 3(b), pode-se escrever as coordenadas x e y do ponto $\mathrm{P}$ em função dos ângulos de orientação $\psi_{1}$ e $\psi_{2}$ como:

$$
\begin{aligned}
& x=X_{f}-n^{*} \operatorname{sen}\left(\psi_{2}\right)^{*} \operatorname{sen}\left(\psi_{1}\right) \\
& y=Y_{f}-n^{*} \operatorname{sen}\left(\psi_{2}\right)^{*} \cos \left(\psi_{1}\right)
\end{aligned}
$$

\section{RESULTADOS E DISCUSSÃO}

Com o intuito de verificar e validar a modelagem matemática estabelecida no item 3, especialmente a combinação entre os modelos matemáticos das duas estruturas que a compõem, foi desenvolvido um programa utilizando o software MATLAB ${ }^{\circledR}$. Assim, a partir dos dados de saída da estrutura robótica paralela combinada (coordenadas cartesianas $x, y$ e $z$ e os ângulos $\psi_{1}, \psi_{2}$ e $\psi_{3}$ de orientação) pode-se encontrar todos os ângulos de entrada das articulações de rotação atuadas (ângulos $\Phi_{1}$ e $\Phi_{2}$ para o mecanismo $5 \mathrm{R}$ e $\theta_{1}, \theta_{2}$ e $\theta_{3}$ para o mecanismo esférico paralelo).

Após a implementação da modelagem matemática, foi desenvolvido o modelo gráfico tridimensional para a estrutura paralela combinada proposta neste trabalho utilizando-se do software SolidWorks ${ }^{\circledR}$. Primeiramente foi modelada a estrutura 5R simétrica, utilizando 5 barras de mesmo comprimento, baseadas nas barras de LEGO ${ }^{\circledR}$, Fig. 5 . 


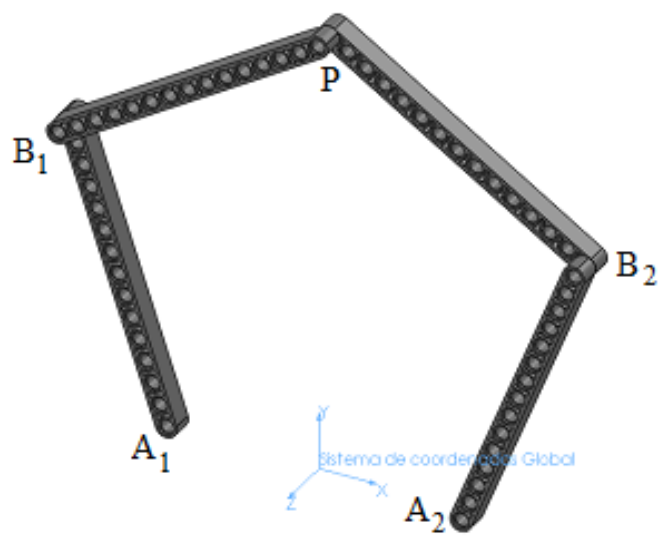

Figura 5. Modelo tridimensional do Mecanismo 5R.

Utilizando este modelo foi possível validar o equacionamento da modelagem matemática para estrutura 5R. Escolhendo posições arbitrárias para o modelo tridimensional e obtendo suas coordenadas quanto ao sistema de coordenadas global, podem-se inserir estas coordenadas no programa de MATLAB ${ }^{\circledR}$ desenvolvido e validar o modelo.

A Tabela 1 apresenta os resultados encontrados para sete posições arbitrárias do modelo computacional.

Tabela 1. Resultados comparativos Modelagem Matemática x Modelo Computacional estrutura 5R.

\begin{tabular}{|c|c|c|c|c|c|c|c|c|}
\hline Posição & $\mathbf{X}(\mathbf{m m})$ & $\mathbf{Y}(\mathbf{m m})$ & $\begin{array}{c}\Phi_{\mathbf{1}} \\
\text { medido } \\
\left({ }^{\circ}\right)\end{array}$ & $\begin{array}{c}\Phi_{\mathbf{2}} \\
\text { medido } \\
\left({ }^{\circ}\right)\end{array}$ & $\begin{array}{c}\Phi_{\mathbf{1}} \\
\text { calculado } \\
\left({ }^{\circ}\right)\end{array}$ & $\begin{array}{c}\Phi_{\mathbf{2}} \\
\text { calculado } \\
\left({ }^{\circ}\right)\end{array}$ & $\begin{array}{c}\text { Erro } \Phi_{\mathbf{1}} \\
(\boldsymbol{\%})\end{array}$ & $\begin{array}{c}\text { Erro } \Phi_{\mathbf{2}} \\
(\boldsymbol{\%})\end{array}$ \\
\hline $\mathbf{1}$ & 272,46 & 442,57 & 83,79 & 23,55 & 83,84 & 23,55 & $0,07 \%$ & $0,01 \%$ \\
\hline $\mathbf{2}$ & $-365,55$ & 671,39 & 141,74 & 125,41 & 141,93 & 126,46 & $0,14 \%$ & $0,83 \%$ \\
\hline $\mathbf{3}$ & 0 & 835,98 & 90,00 & 90,00 & 90,21 & 89,78 & $0,24 \%$ & $-0,24 \%$ \\
\hline $\mathbf{4}$ & 0 & 496,24 & 118,33 & 61,76 & 118,29 & 61,70 & $-0,03 \%$ & $-0,08 \%$ \\
\hline $\mathbf{5}$ & 0 & 387,98 & 120,00 & 60,00 & 119,99 & 60,00 & $0,00 \%$ & $0,00 \%$ \\
\hline $\mathbf{6}$ & $-663,88$ & 120,37 & $-135,90$ & 172,33 & $-135,81$ & 169,69 & $0,06 \%$ & $-1,55 \%$ \\
\hline $\mathbf{7}$ & 666,47 & 99,26 & 6,64 & $-46,95$ & 6,86 & $-47,09$ & $3,21 \%$ & $0,13 \%$ \\
\hline
\end{tabular}

As posições 6 e 7 da Tab. 1 apresentaram maiores valores de erro devido à proximidade do posicionamento com configurações de singularidade do mecanismo 5R, Fig. 6.

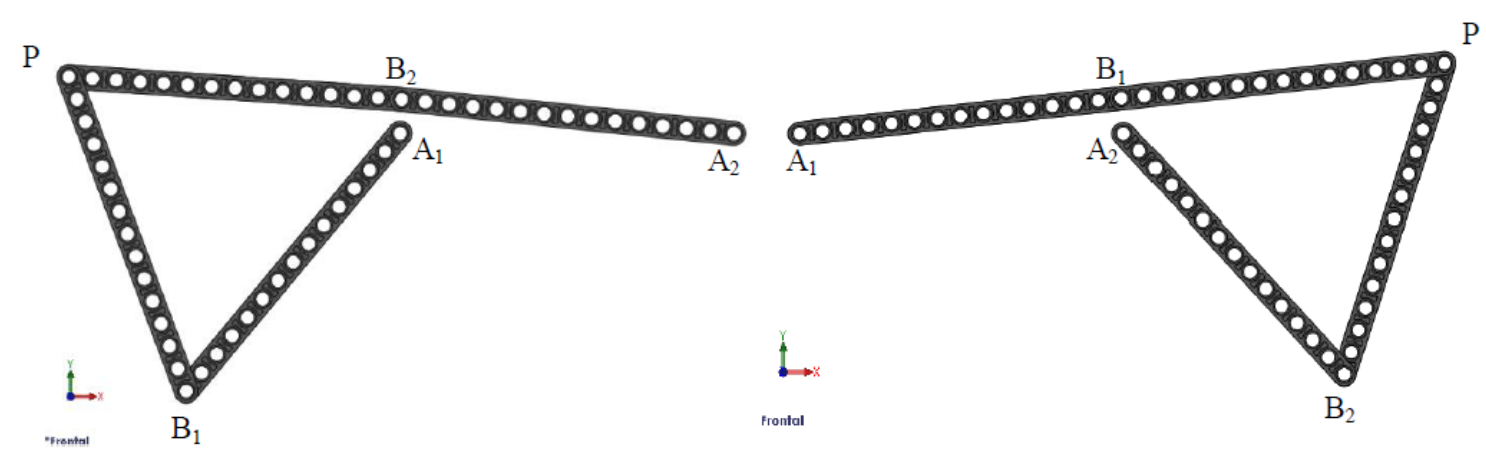

(a)

(b)

Figura 6. Posições de singularidade do mecanismo 5R. (a) Posição 6 da Tab. 1; (b) Posição 7 da Tab. 1.

Em seguida, foi desenvolvido o modelo gráfico tridimensional do mecanismo esférico paralelo, tomando como base o Agile Eye. Conforme já comentado no item 3, de maneira a melhorar a performance geral do mecanismo, o mesmo foi construído com dois segmentos rígidos, acoplados num ângulo de $90^{\circ}$, formando o link proximal, e um segmento único para o link distal. Os modelos construídos para estes dois segmentos são mostrados separadamente nas Figs. 7(a) e 7(b). 


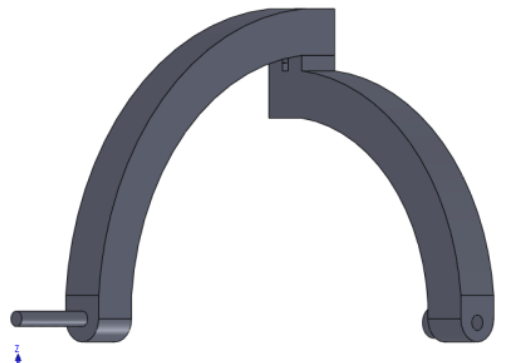

L.

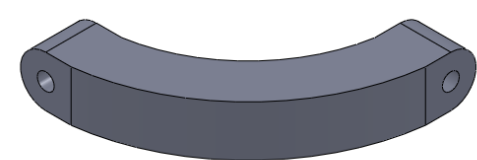

i.

(a)

(b)

Figura 7. Links do mecanismo esférico paralelo. (a) Link proximal; (b) Link distal.

Tanto os segmentos, quanto a base e o atuador foram construídos seguindo as premissas expostas por Gosselin et al. (1994) para o manipulador: o ângulo entre cada articulação da base (vetores u) deve ser $90^{\circ}$, assim como o ângulo entre a articulação da base e a articulação subsequente entre os links proximal e distal (vetores w) de cada segmento também deve ser $90^{\circ}$ e todos os vetores das articulações devem apontar para o centro de rotação do mecanismo, resultando assim em um mecanismo simétrico.

A estrutura paralela esférica é apresentada na Fig. 8, onde se pode ver a estrutura em sua posição inicial Fig. 8(a) e o centro de rotação da estrutura Fig. 8(b).

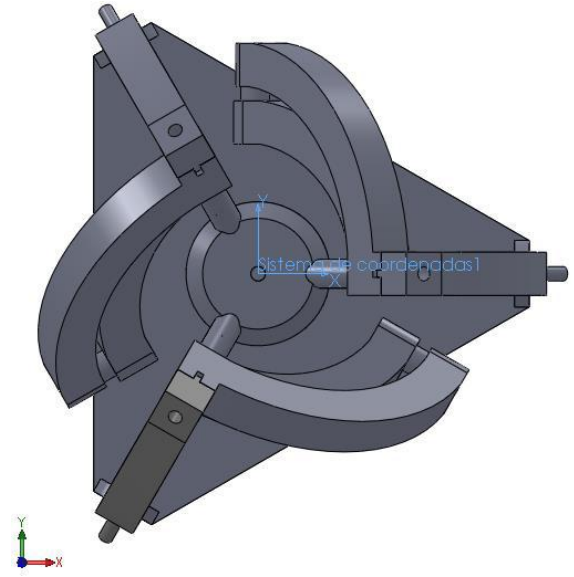

(a)

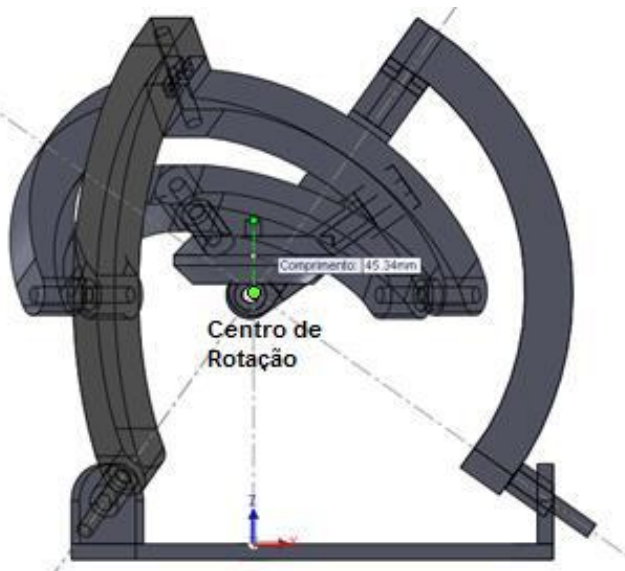

(b)

Figura 8. Modelo do Mecanismo Esférico Paralelo. (a) posição inicial; (b) centro de rotação do mecanismo.

A validação do modelo matemático, assim como foi feita para o mecanismo $5 \mathrm{R}$, no caso do mecanismo esférico paralelo foi feita juntamente com a validação da combinação das estruturas, uma vez que são idênticas para a estrutura isolada e para a estrutura combinada. Tal combinação foi feita através do ponto central da base da estrutura esférica. A combinação foi feita de tal maneira que não permita que a estrutura gire em torno deste ponto, uma vez que esse movimento acrescentaria mais uma rotação à estrutura combinada. $\mathrm{O}$ modelo da estrutura combinada pode ser visto na Fig. 9(a). Na Figura 9(b) pode-se ver o acoplamento entre as duas estruturas.

Seguindo o mesmo procedimento do mecanismo 5R, agora com o modelo gráfico tridimensional do Mecanismo Paralelo Combinado, posiciona-se o atuador do mecanismo em diferentes pontos do espaço, e mede-se a partir do sistema de coordenadas global para os parâmetros de saída do mecanismo (os ângulos de orientação $\psi_{1}$, $\psi_{2}$ e $\psi_{3}$ e coordenadas de posição $x, y$ e $z$ ). Utilizam-se estes dados no programa do modelo matemático e compara-se com os valores medidos no modelo gráfico tridimensional. A Tabela 2 mostra os dados de entrada para uma dada posição do mecanismo, com duas orientações diferentes.

Tabela 2. Dados de entrada para testes realizados com o modelo tridimensional.

\begin{tabular}{|l|l|l|l|l|l|l|}
\hline Posição & Orientação & $\psi_{1}\left({ }^{\circ}\right)$ & $\psi_{2}\left(^{\circ}\right)$ & $\psi_{3}\left(^{\circ}\right)$ & $\mathbf{X}(\mathbf{m m})$ & Y (mm) \\
\hline $\mathbf{1}$ & $\mathbf{1}$ & 0,00 & 12,74 & 0,00 & 217,27 & 587,37 \\
\hline $\mathbf{1}$ & $\mathbf{2}$ & 38,66 & 25,72 & 0,00 & 221,43 & 602,29 \\
\hline
\end{tabular}




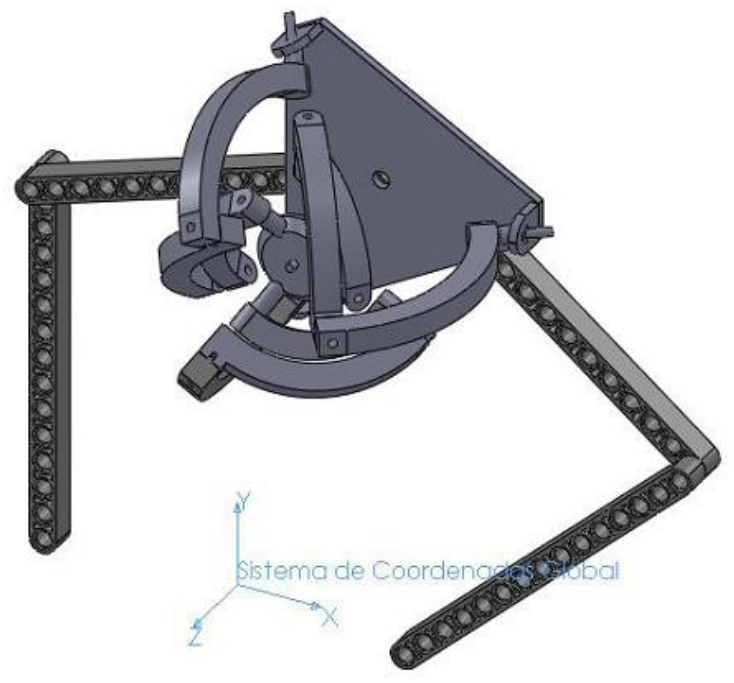

(a)

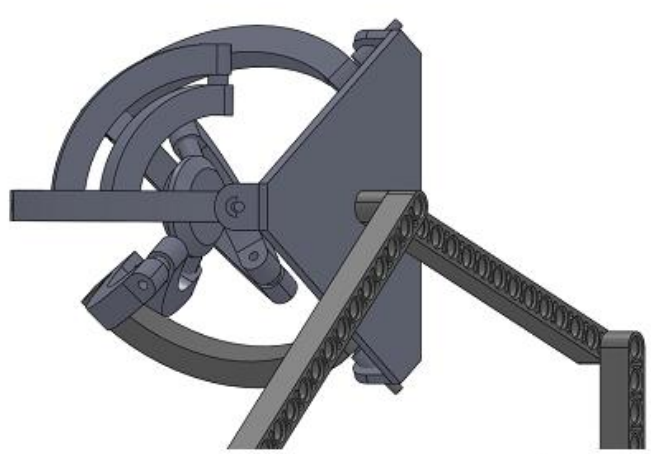

(b)

Figura 9. (a) Vista Trimétrica do Manipulador Paralelo Combinado; (b) Acoplamento entre as duas estruturas.

As tabelas 3 e 4 por sua vez trazem os resultados da comparação dos ângulos $\theta$ para a orientação (mecanismo esférico) e para a posição (mecanismo 5R) respectivamente. Dos resultados apresentados os modelos são semelhantes.

Tabela 3. Resultados para orientação do mecanismo combinado (ângulos $\theta$ do mecanismo esférico).

\begin{tabular}{|c|c|c|c|c|c|c|c|c|c|}
\hline Orientação & $\begin{array}{c}\theta_{1}\left({ }^{\circ}\right) \\
\text { medido }\end{array}$ & $\begin{array}{c}\theta_{2}\left({ }^{\circ}\right) \\
\text { medido }\end{array}$ & $\begin{array}{c}\theta_{3}\left({ }^{\circ}\right) \\
\text { medido }\end{array}$ & $\begin{array}{c}\theta_{1}\left({ }^{\circ}\right) \\
\text { calculado }\end{array}$ & $\begin{array}{c}\theta_{2}\left({ }^{\circ}\right) \\
\text { calculado }\end{array}$ & $\begin{array}{c}\theta_{3}\left({ }^{\circ}\right) \\
\text { calculado }\end{array}$ & $\begin{array}{c}\text { Erro } \theta_{1} \\
(\%)\end{array}$ & $\begin{array}{c}\text { Erro } \theta_{2} \\
(\%)\end{array}$ & $\begin{array}{c}\text { Erro } \theta_{3} \\
(\%)\end{array}$ \\
\hline 1 & 90,00 & 95,64 & 84,36 & 90,00 & 97,03 & 82,97 & $0,0 \%$ & $1,5 \%$ & $1,6 \%$ \\
\hline 2 & 82,08 & 103,62 & 83,64 & 81,18 & 105,10 & 83,17 & $1,1 \%$ & $1,4 \%$ & $0,6 \%$ \\
\hline
\end{tabular}

Tabela 4. Resultados da posição do mecanismo combinado (ângulos $\Phi$ do mecanismo 5R).

\begin{tabular}{|l|l|l|l|l|l|l|}
\hline Orientação & $\begin{array}{l}\Phi_{\mathbf{1}} \text { medido } \\
\left({ }^{\circ}\right)\end{array}$ & $\begin{array}{l}\Phi_{\mathbf{2}} \text { medido } \\
\left({ }^{\circ}\right)\end{array}$ & $\begin{array}{l}\Phi_{\mathbf{1}} \text { calculado } \\
\left({ }^{\circ}\right)\end{array}$ & $\begin{array}{l}\Phi_{\mathbf{2}} \text { calculado } \\
\left({ }^{\circ}\right)\end{array}$ & Erro $\boldsymbol{\Phi}_{\mathbf{1}}(\boldsymbol{\%})$ & Erro $_{\mathbf{2}}(\boldsymbol{\%})$ \\
\hline $\mathbf{1}$ & 89,98 & 43,09 & 88,41 & 41,79 & $1,7 \%$ & $3,0 \%$ \\
\hline $\mathbf{2}$ & 89,98 & 43,09 & 89,08 & 42,39 & $1,0 \%$ & $1,6 \%$ \\
\hline
\end{tabular}

\section{CONCLUSÃO}

Este trabalho se propôs a estudar as estruturas paralelas combinadas formadas por vários mecanismos paralelos, colocados em sequência. As estruturas paralelas têm algumas vantagens sobre as estruturas seriais, porém sofrem com um espaço de trabalho limitado e maior complexidade na resolução da cadeia cinemática.

Uma nova estrutura paralela combinada foi proposta a partir da combinação dos mecanismos 5R paralelo plano e esférico paralelo, este último sob os moldes de uma estrutura já existente, o Agile Eye.

Os modelos matemáticos das estruturas individuais foram calculados. As estruturas foram modeladas tridimensionalmente e combinadas para dar origem à estrutura proposta. Por fim, os modelos matemático e gráfico foram confrontados um com o outro, e seus resultados avaliados.

Os valores de erro para a posição confirmam a validade do modelo matemático utilizado para o mecanismo 5R, assim como os valores de erro para orientação confirmam a validade do modelo utilizado para o mecanismo esférico paralelo. Os resultados de posição, utilizando as coordenadas $x$ e $y$ do elemento terminal do mecanismo esférico paralelo, por sua vez, validam as equações utilizadas para combinação dos mecanismos.

Como trabalhos futuro estão previstos a análise de rigidez da estrutura e a construção da estrutura composta proposta.

\section{AGRADECIMENTOS}

Os autores agradecem a UFU, FEMEC, FAPEMIG, CAPES e CNPQ pelo apoio financeiro parcial a este trabalho.

\section{REFERÊNCIAS}


Badescu, M.; Mavroidis, C. New Performance Indices and Workspace Analysis of Reconfigurable Hyper-Redundant Robotic Arms. The International Journal of Robotics Research, v. 23, n. 6, p. 643-659, 2004.

Badescu, M.; Mavroidis, C.; Morman, J. Workspace Optimization of Orientational 3-Legged UPS Parallel Platforms. Proceedings of the 2002 ASME International Design Engineering Technical Conferences and the Computers and Information in Engineering Conference (DETC/CIE), 2002.

Bai, S. Optimum design of spherical parallel manipulators for a prescribed workspace, Mechanism and Machine Theory 45, p. 200-211, 2010.

Bai, S., Hansen, M.R., Angeles, J. A robust forward-displacement analysis of spherical parallel robots, Mechanism and Machine Theory 44, p. 2204-2216, 2009

Gonçalves, R. S. Estudo De Rigidez De Cadeias Cinemáticas Fechadas. 2009. 239 (Tese de Doutorado). Universidade Federal de Uberlândia.

Gosselin, C.M., Hamel, J.-F. The agile eye: a high-performance three-degree-of-freedom camera-orienting device, IEEE International Conference on Robotics and Automation, 1994

Gosselin, C.M., Pierre, E.S., Gagne, M. On the Development of the Agile Eye, IEEE Robotics \&Automation Magazine 29, 1996.

Li, T., Payandeh, S. Design of spherical parallel mechanisms for application to laparoscopic surgery, Robotica 20, p.133-138, 2002.

Liu, X.-J., Wang, J., Pritschow, G. Kinematics, singularity and workspace of planar 5R symmetrical parallel mechanisms, Mechanism and Machine Theory 41, p. 145-169, 2006a.

Ramadan, A. A., Takubo, T., Mae, Y., Ohara, K., Arai, T. Developmental Process of a Chopstick-Like Hybrid-Structure Two-Fingered Micromanipulator Hand for 3-D Manipulation of Microscopic Objects. IEEE Transactions on Industrial Electronics v. 56, n. 4, p. 1121-1135, 2009.

Tanev, T. K. Workspace of a Hybrid (Parallel-Serial) Robot Manipulator. Problems Of Engineering Cybernetics And Robotics, v. 56, 2006.

Wu, H.; Handroos, H.; Pessi, P. A Hybrid Parallel Robot. Conference on Robotics, Automation and Mechatronics, p. 640-644, 2008.

Yabugaki, H., Ohara, K., Kojima, M., Mae, Y., Tanikawa, T., Arai, T. Automated Stable Grasping with Two-Fingered Microhand using Micro Force Sensor, IEEE International Conference on Robotics and Automation (ICRA), 2013.

Yoon, J.; Ryu, J. A New Family of Hybrid 4-dof Parallel Mechanisms with Two Platforms and its Application to a Footpad Device. Journal of Robotic Systems, v. 22, n. 5, p. 287-298, 2005.

Zhao, W., Li, B., Yu, H., Hu, Y. Study on a Novel 6-DOF Combinational Parallel Manipulator, Proceedings of the 2008 IEEE International Conference on Robotics and Biomimetics, Bangkok, Thailand, 2009.

Zheng, X. Z.; Bin, H. Z.; Luo, Y. G. Kinematic Analysis of a Hybrid Serial-Parallel Manipulator. International Journal of Advanced Manufacturing Technology, v. 23, p. 925-930, 2004.

\title{
RESPONSABILIDADE AUTORAL
}

“Os autores são os únicos responsáveis pelo conteúdo deste trabalho".

\section{STUDY OF A NEW PARALLEL ROBOTIC STRUCTURE COMBINED}

\author{
João Marcus Teixeira Pêgo, j.marcus.gtr7@hotmail.com ${ }^{1}$ \\ João Carlos Mendes Carvalho, jcmendes@mecanica.ufu.br ${ }^{1}$ \\ Rogério Sales Gonçalves, rsgoncalves@mecanica.ufu.br ${ }^{1}$
}

${ }^{1}$ Universidade Federal de Uberlândia - Faculdade de Engenharia Mecânica - Av. João Naves de Ávila, 2121, Bairro Santa Mônica, Uberlândia - MG - CEP 38400-902

\begin{abstract}
Robotic structures are commonly divided into serial, parallel and hybrid structures. The serial structure consists of an end-effector attached to a base through a single kinematic chain. A parallel structure on the other hand can be described as a mechanism composed of an end-effector connected to a fixed base through two or more kinematic chains. Parallel structures can have some advantages over serial structures, such as better stiffness and higher carry load, but suffer with a limited workspace and a greater complexity in their mathematical modeling. The third type of structure is the hybrid structures, combinations of serial and parallel structures. These can also consist of a combination between more than one parallel structure. This paper's objective is to study the combined parallel structures composed by multiple parallel mechanisms. Starting with a review on the hybrid structures, composed by parallel structures, in this paper a new structure is proposed. It is formed by the 5R symmetrical parallel mechanism and the spherical parallel mechanism. The mathematical modeling of this new structure is calculated through the individual models of the structures that compose it. Finally, a tridimensional graphic model of the new structure was developed and confronted with the mathematical model.
\end{abstract}

Keywords: Robotic Structures, 5R Planar Mechanism, Spherical Parallel Mechanism. 